\title{
Echange ionique entre une couche mince obtenue par procédé sol-gel et un bain de $\mathrm{CuCl}$ fondu
}

\author{
A. RAADA, J. ZARZYCKI, C. PARENT* et G. LE FLEM ${ }^{*}$ \\ Laboratoire de Science des Matériaux Vitreux, U.A. 1119, CNRS Université de Montpellier II, Place E. \\ Bataillon, 34095 Montpellier Cedex 5, France \\ ${ }^{*}$ Laboratoire de Chimie du Solide, CNRS 351, Cours de la Libération, 33405 Talence, France
}

\section{Résumé}

L'échange ionique entre une couche mince de verre obtenue par procédé sol-gel ( déposée sur une lame de verre à vitre par la technique de dip-coating), et un bain de $\mathrm{CuCl}$ fondu à $525^{\circ} \mathrm{C}$ et sous atmosphère d'azote a été étudié pour trois séries de verres aluminosilicates $\mathrm{SiO}_{2}-\mathrm{Al}_{2} \mathrm{O}_{3}-\mathrm{X}_{2} \mathrm{O}(\mathrm{X}=\mathrm{Na}, \mathrm{K}, \mathrm{Li})$. L'expérience montre l'existence des ions $\mathrm{Cu}+$ monovalents introduits dans le verre.

\section{INTRODUCTION}

Le cuivre monovalent dans les verres donne naissance à une intense luminescence [1-3]. Ces matériaux sont envisagés comme luminophores ou comme sources pour lasers accordables. Par ailleurs, la possibilité d'introduire $\mathrm{Cu}+$ dans le verre par échanges ioniques entre un sel fondu et un verre a été montrée par plusieurs auteurs [4-10].

Dans le travail présenté ici, nous avons tenté d'utiliser pour cela des couches minces de verre obtenues par le procédé sol-gel, ce qui n'avait jamais été fait jusqu'à prèsent, à notre connaissance.

\section{METHODES EXPERIMENTALES}

\section{1-Préparation des solutions pour dip-coating}

Les couches minces de verre ont été elaborées par hydrolyse et polycondensation des composés organométalliques, [11-12]. Le tétraéthoxysilane $\left.\mathrm{Si}_{(\mathrm{OC}} \mathrm{H}_{5}\right)_{4}$ et l'aluminium-sec-butoxide Al(OCH[CH $\left.]_{3} \mathrm{C}_{2} \mathrm{H}_{5}\right)_{3}$ ont été utilisés comme précurseures pour la préparation des 3 solutions de composition: TOSiO $_{2}-10 \mathrm{Al}_{2} \mathrm{O}_{3}-20 \mathrm{X}_{2} \mathrm{O}$, ( en mol\% ), avec $\mathrm{X}=\mathrm{Na}, \mathrm{K}$, ou Li. L'organigramme de la préparation est donné sur la Figure 1 .

\section{2- Réalisation des couches minces}

La technique utilisée est celle du "dip coating", les substrats étant des lames de verre à vitre pour préparations microscopiques.

a- Procédé de nettoyage du substrat

Pour éliminer toute trace d'agent polluant et avoir une meilleure adhésion de la couche sur le substrat, la procédure suivante a été suivie: nettoyage des substrats à l'aide d'un mélange sulfochromique à $70^{\circ} \mathrm{C}$ pendant 2 heures, puis traitement aux ultrasons, rinçage à l'eau déminéralisée et à l'ethanol. b- Tirage

Pour des solutions de concentration de $10 \mathrm{~g} / \mathrm{l}$, et à des taux d'humidité ambiante de $40 \%$ environ, on a utilisé des vitesses de tirage de l'ordre de $120 \mathrm{~mm} / \mathrm{mn}$.

c- Hydrolyse

Cette réaction se fait aprés chaque tirage par l'humidité de l'air à température ambiante. 
d- Traitement thermique des couches

La transformation en un film d'oxyde se fait a l'air dans un four a $500^{\circ} \mathrm{C}$ pendant 20 minutes, après un dépót de 5 couches; cette opération est répétée 4 fois.

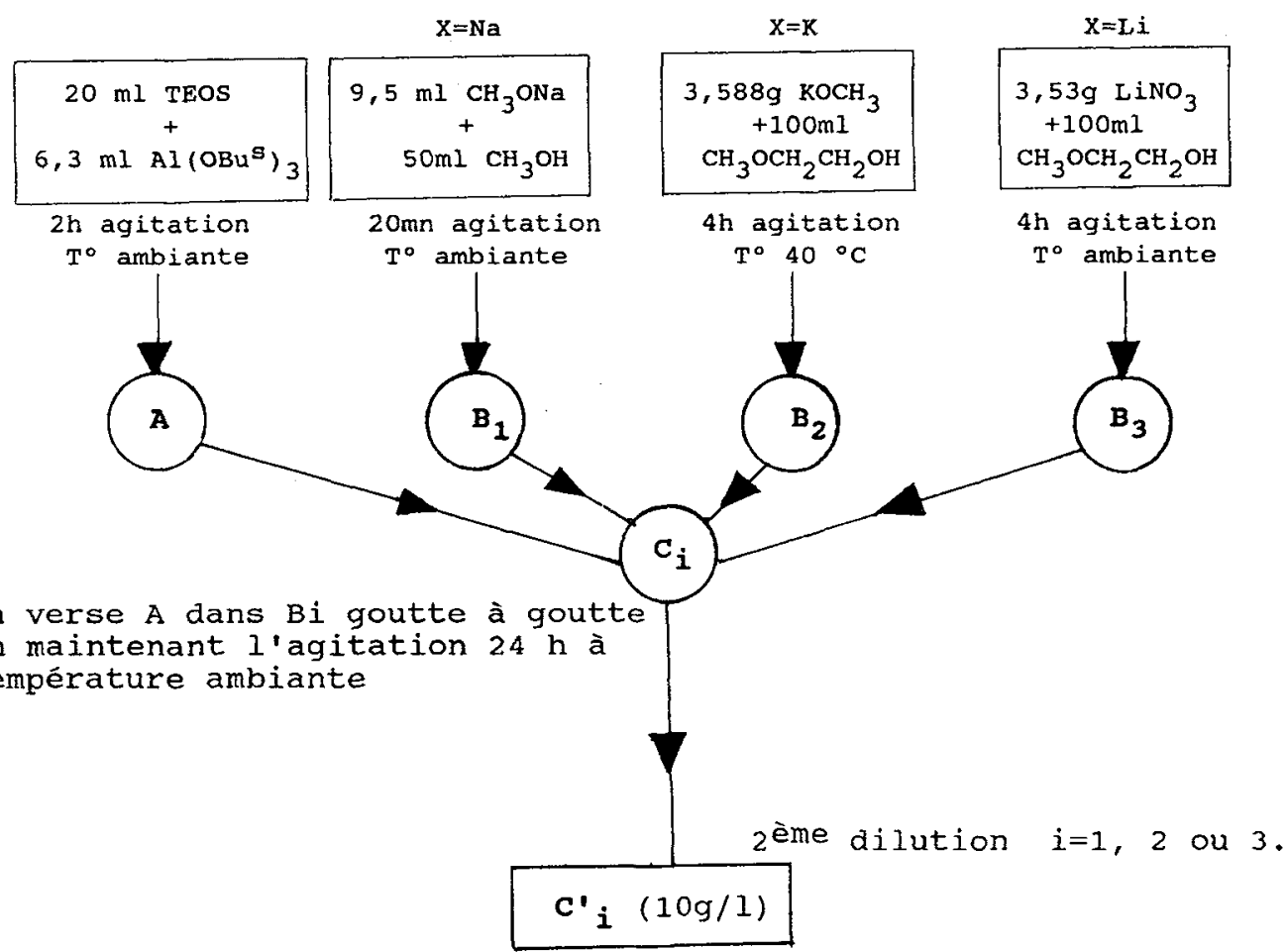

Fig. 1: Organigramme de la préparation des solutions pour dip-coating

\section{3-Echange ionique}

Le montage utilisé est constitué par un ballon en silice vitreuse avec deux tubulures;une tige en cuivre tient l'échantilion en lui permettant un déplacement vertical à l'interieur du ballon où est placé un creuset en silice contenant $\mathrm{CuCl}$, une pompe à vide sert a évacuer l'air, et l'ensemble est placé à l'intérieur d'un four, couplé à un régulateur de température. On réalise à l'interieur du ballon un vide à $200^{\circ} \mathrm{C}$ pendant 60 minutes. Aprés avoir introduit l'azote à une pression d'une atmosphère et stabilisé la température à $525^{\circ} \mathrm{C}$ on plonge l'échantillon dans le bain de $\mathrm{CuCl}$ fondu pendant les temps suivants : 15, 30,60 ou 100 minutes.

\section{4- Caractérisation}

L'étude de luminescence, émission et excitation, a été réalisée à l'aide d'un spectrofluorimètre JOBIN-YVON, au Laboratoire de Chimie de Solide à Talence.

La source lumineuse est une lampe arc de $150 \mathrm{~W}$ concentré dans une atmosphère de xénon a haute pression. Cette source émet dans un domaine de longueur d'onde entre $200 \mathrm{~nm}$ et $1000 \mathrm{~nm}$.

Le monochromateur d'excitation est constitué par un prisme en quartz. La répartition spectrale des radiations émises par l'échantilion est analysée par un monochromateur d'analyse (JOBIN-YVON HRS2). Le flux lumineux qui sort du monochromateur $d$ 'analyse est détecté par un photomultiplicateur (HAMAMATSU R929). 
Ce spectrofluorimètre permet soit d'effectuer l'étude spectrale de la lumière émise par un échantillon soumis a une radiation de longueur d'onde fixe (spectre dit d'émission ou de fluorescence), soit de mesurer l'intensité de la lumière émise à une longueur d'onde fixe par un échantillon excité par une radiation de longueur d'onde variable (spectre d'excitation).

Les spectres obtenus ont été corrigés à l'aide d'un compteur de photons et d'un microprocesseur (Intel D 8253-) relié à un ordinateur IBM.

Le XPS à été également utilisé parmi les méthodes de caractérisation. Les spectres ont été enregistrés sur un spectromètre de photoéléctrons (AEl type ES 200B). Le rayonnement $X$ excitateur est fourni par une anticathode de magnésium $\left(\mathrm{MgK}_{\alpha}\right)$, pour une energie de $1253,6 \mathrm{eV}$.

\section{RESULTATS}

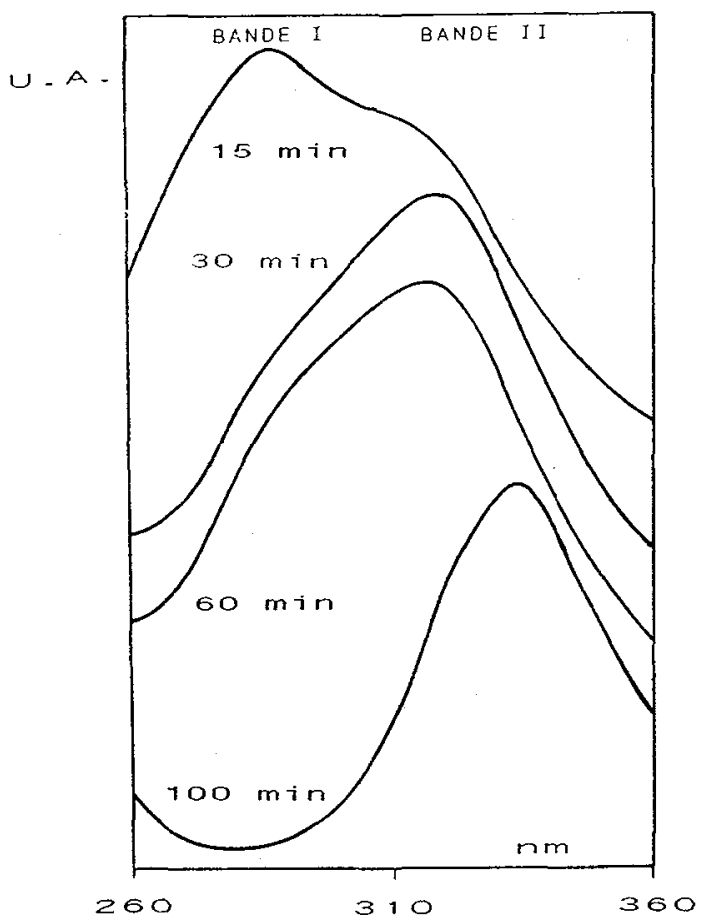

SPECTRE D'EXCITATION ( $\left.T=300 \mathrm{~K} ; \lambda_{0 .}=550 \mathrm{~nm}\right)$

Bande I: caractéristique de substrat.

Bande II: caractéristique de la couche de verre.

1- Emission et excitation de la luminescence de $\mathrm{Cu}^{+}$dans les lames échangées sans couches (lames temoins):ces lames présentent toutes la même luminescence;à 300K et sous excitation de $320 \mathrm{~nm}$ on observe une bande d'émission centrée sur $540 \mathrm{~nm}$ et s'étendant dans tout le domaine de visible entre 400 et $650 \mathrm{~nm}$ et une bande d'excitation située entre 230 et $360 \mathrm{~nm}$ avec un maximum vers $290 \mathrm{~nm}$ à $300 \mathrm{~K}$ pour $\lambda_{\mathrm{em}}=550 \mathrm{~nm}$.

2- Emission et excitation de $\mathrm{Cu}^{+}$dans les lames de verre avec une couche mince de $\mathrm{SiO}_{2}-\mathrm{Al}_{2} \mathrm{O}_{3}$ $\mathrm{Na}_{2} \mathrm{O}$. Le spectre d'excitation d'un échantillon qui à subi 15 minutes d'échange, d $300 \mathrm{~K}$ pour $\lambda_{\text {em }}=550 \mathrm{~nm}$ comporte deux bandes;une caractéristique de la lame (substrat) centrée sur $285 \mathrm{~nm}$ et une autre bande caractéristique de la couche vers $320 \mathrm{~nm}$ avec une intensité plus faible que celle de la première. 
Lorsque le temps d'échange ionique augmente de 30 a 100 minutes, l'intensité de la première bande diminue par rapport à celle de la deuxième. A 100 minutes d'échange seuls les ions cuivre introduits dans la couche sont détéctables, (Figure 2).

Pour les spectres d'émission effectués à $300 \mathrm{~K}$ et sous excitation de $320 \mathrm{~nm}$, on ne voit qu 'une seule bande large entre 400 et $650 \mathrm{~nm}$ avec un maximum à $535 \mathrm{~nm}$.

3- Emission et excitation de $\mathrm{Cu}^{+}$dans les lames de verre avec une couche mince de $\mathrm{SiO}_{2}-\mathrm{Al}_{2} \mathrm{O}_{3}-$ $\mathrm{K}_{2} \mathrm{O}$. Le spectre d'émission d'un échantillon qui a été échangé pendant 15 minutes, à $300 \mathrm{~K}$ et sous excitation de $320 \mathrm{~nm}$ présente deux bandes; une bande caractéristique de la lame avec un maximum à 550 $\mathrm{nm}$ et une autre bande caractéristique de la couche avec un maximum a $675 \mathrm{~nm}$.

Le spectre d'excitation à $300 \mathrm{~K}$ et pour $\lambda_{\mathrm{em}}=550 \mathrm{~nm}$ ne présente une seule bande large entre 260 et 440 $\mathrm{nm}$ avec un maximum à $330 \mathrm{~nm}$.

4- Emission et excitation de $\mathrm{Cu}+$ dans les lames de verre avec une couche mince de $\mathrm{SiO}_{2}-\mathrm{Al}_{2} \mathrm{O}_{3}$ $\mathrm{Li}_{2} \mathrm{O}$. La luminescence de cet échantillon ne présente qu'une seule bande large entre 400 et $760 \mathrm{~nm}$ avec un maximum à $600 \mathrm{~nm}$ pour l'émission à $300 \mathrm{~K}$ et sous excitation de $320 \mathrm{~nm}$. Le spectre d'excitation à $300 \mathrm{~K}$ et pour $\lambda_{\mathrm{em}}=550 \mathrm{~nm}$ ne présente qu'une bande entre 260 et $360 \mathrm{~nm}$ centrée sur $310 \mathrm{~nm}$.

\section{Caractérisation de l'élement cuivre par XPS}

Les études de spectres XPS relatifs aux différentes états d'oxydation de cuivre ont montré que le cuivre monovalent est caractérisé par un spectre présentant deux pics $2 p$ : un pic $2 p^{3 / 2}$ a $932 e V$ et un pic $2 p^{1 / 2}$ a $952 \mathrm{eV}$, alors que le cuivre divalent est caractérisé par un spectre présentant deux pics et deux satellites: un pic $2 \mathrm{p}^{3 / 2}$ a $933,6 \mathrm{eV}$, un pic $2 \mathrm{p}^{1 / 2}$ a $953,6 \mathrm{eV}$, avec deux satellites, un centré sur $942 \mathrm{eV}$ et l'autre sur $962 \mathrm{eV}$ [13]. Les spectres obtenus pour tous les échantillons échangés présentent des pics $2 \mathrm{p}^{3 / 2}$ vers $932 \mathrm{eV}$ sans satellites.

\section{DISCUSSION}

L'existence de deux bandes dans les spectres d'excitation ou d'émission pour les séries $\mathrm{SiO}_{2}$ $\mathrm{Al}_{2} \mathrm{O}_{3}-\mathrm{Na}_{2} \mathrm{O}$ et $\mathrm{SiO}_{2}-\mathrm{Al}_{2} \mathrm{O}_{3}-\mathrm{K}_{2} \mathrm{O}$ a permis de distinguer deux familles de sites de $\mathrm{Cu}^{+}$: une famille correspondant à la couche deposée et l'autre correspondant à la lame (substrat). L'intérpretation de ces résultats peut-être la suivante:au début de l'échange les ions $\mathrm{Cu}{ }^{+}$traversent la couche et vont se fixer majoritairement sur la lame, une plus faible proportion étant piégée sur la couche. Au bout d'un certain temps d'échange ( 30 à $60 \mathrm{~min}$ ), cette tendance s'inverse, peut-être en raison d'une saturation en cuivre de la surface de la lame. Enfin, dans le cas d'echange pendant $100 \mathrm{~min}$, seuls les ions cuivre introduits dans la couche sont détectés d'une part en raison de leur plus grande proportion mais également par suite d'un "masquage" des centres luminescents de la lame support.

Ces conclusions ont pu être tirées grâce à une identification précise des spectres relatifs à chacun des centres $\mathrm{Cu}^{+}$dans les substrats et dans les couches.

L'absence de satellites et l'énergie de liaison d'environ 931-932 eV dans les spectres XPS. suggèrent qu'il s'agit bien de cuivre monovalent.

\section{REFERENCES}

[1] A.R.RODRIGUEZ, C.W.PARMENLBEE et A.E.BADGER -J.Am.Ceram.Soc.,26 (5), 137, 1943.

[2] J.C.ZHANG,B.MOINE, C.PEDRINI,C.PARENT, et G.LE FLEM - J.Phys.Chem.Solids 51 (1990) 933.

[3] P.BOUTINAUD, E.DULOISY,C.PEDRINI,B.MOINE,C.PARENT,et G.LE FLEM -J.Solid State Chem. 94 (1991) 236.

[4] J.L.BARTON et DE BILLY - J. Non. Cryst. Sol. 38 et 39 (1980) 523-526.

[5] S.SAKKA, K.KAMIYA et K.KATO - J. Non. Cryst. Sol. 52 (1982) 77-90.

[6] T.YOKO, KAMIYA et S.SAKKA - Revue de Chimie Minérale .10,1983,p.668.

[7] T.YOKO, KAMIYA et S.SAKKA - J. Non. Cryst. Sol. 71 (1985) 245-251.

[8] R.DEBNATH et S.KUMAR - J. Non. Cryst. Sol. 123 (1990) 271-274.

[9] T.YOKO,T.NISHIWAKI, K.KAMIA et S.SAKKA - J.Am.Ceram.Soc. 74 [5] 1104-11 (1991).

[10] NIGAMP.RATH, ELIZABETHM.HOLT, et KATSUMI TANIMURA - Inorg.Chem. 1985,24,3934-3938.

[11] J.PHALIPPOU, M.PRASSAS et J.ZARZYCKI - Verres et Refract., Vol.35, nº6, Nov-Dec, (1981) 975981

[12] H.DISLICH et E.HUSSMANN, - Thin Solids Films, 77(1981)129-139.

[13] Z.HUSSAIN, M.A.SALIM, M.A.KHAN et E.E.KHAWAJA.- J. Non.Cryst. Sol. 110 (1989) 44-52. 